\title{
Synergistic effect of In doping on electrical and thermal properties of $\mathrm{Cu}_{2} \mathrm{SnSe}_{3}$ thermoelectric system
}

\author{
Riya Thomas ${ }^{1}$, Ashok Rao ${ }^{1, *}$ (1), Zhao-Ze Jiang ${ }^{2}$, and Yung-Kang Kuo ${ }^{2, *}$ \\ ${ }^{1}$ Department of Physics, Manipal Institute of Technology, Manipal Academy of Higher Education, Manipal 576104, India \\ ${ }^{2}$ Department of Physics, National Dong Hwa University, Hualien 97401, Taiwan
}

Received: 14 October 2020

Accepted: 27 January 2021

(C) The Author(s) 2021

\begin{abstract}
$\mathrm{Cu}_{2} \mathrm{SnSe}_{3}$ has been considered as a potential thermoelectric material owing to its tunable transport properties and its phonon-glass-electron-crystal (PGEC) characteristics. Here, $p$-type pure and In-doped $\mathrm{Cu}_{2} \mathrm{SnSe}_{3}$ samples are synthesized by the solid-state sintering technique. Cubic structure with $F \overline{4} 3 m$ space group is maintained for all the samples, and a linear increase in lattice parameter with increasing In concentration has been observed. The nature of electrical resistivity changes from semiconducting to metallic behavior for samples with $x>0.10$. The decrease in both electrical resistivity and Seebeck coefficient with an increase in $x$ is attributed to the increased hole concentration. Such a scenario is confirmed from the room-temperature Hall effect measurements. Indium doping also reduces the thermal conductivity of the $\mathrm{Cu}_{2} \mathrm{SnSe}_{3}$ system as a result of increased phonon scattering due to the mass fluctuation. Concurrently, enhancement of thermoelectric power factor $(P F)$ and figure of merit $(Z T)$ is achieved with In doping at $\mathrm{Sn}$ site of $\mathrm{Cu}_{2} \mathrm{SnSe}_{3}$. The maximum $\mathrm{ZT}$ of 0.04 has been exhibited by the sample with $x=0.25$ at $400 \mathrm{~K}$, which is six times higher than that of the undoped $\mathrm{Cu}_{2} \mathrm{SnSe}_{3}$.
\end{abstract}

\section{Introduction}

The search for environment friendly and renewable energy sources is on the rise and could hold the key to combating issues like climate change, dwindling fossil fuel reserves, and growing global energy demands. Thermoelectric (TE) technology, which directly interconverts electrical and thermal energy based on the well-known thermoelectric effects (Seebeck and Peltier effects), can be utilized as a pollution-free means of power generation and refrigeration [1-4]. However, the adoption of TE devices is not widespread due to its insufficient conversion efficiency. The performance of a TE device is determined by the materials transport properties, namely the electrical conductivity $(\sigma)$, the Seebeck coefficient $(S)$, and thermal conductivity $(\kappa)$, where thermal conductivity is a sum of lattice and electronic contributions $\left(\kappa_{L}+\kappa_{e}\right)$. The efficiency of TE materials at absolute temperature $T$ is gauged by the dimensionless TE figure of merit, $Z T=\frac{S^{2} \sigma}{\kappa} T[1,2]$.

Address correspondence to E-mail: ashokanu_rao@rediffmail.com; ykkuo@gms.ndhu.edu.tw 
The simultaneous enhancement of electrical conductivity and the Seebeck coefficient, along with the reduction in thermal conductivity, is a difficult challenge as these physical quantities are correlated and usually conflict with each other [2, 3]. This hinders the use of TE materials for commercial applications. Thus, in the last few years, efforts have been made to attain high $Z T$ value by utilizing different classes of materials like skutterudites, clathrates, complex chalcogenides, $\mathrm{Bi}_{2} \mathrm{Te}_{3}$, half-Heuslers, etc., and by employing various strategies like elemental doping, composite engineering, alloying, and nanostructuring [5-10].

The Cu-based multinary chalcogenides have recently attained a lot of consideration as promising TE materials because of their tunable transport properties. Besides, this class of materials has the advantages of low toxicity and high elemental abundance and therefore are considered to be ecofriendly and cost-effective candidates for TE applications [10-12]. Among the Cu-based thermoelectric materials, the diamond-like ternary selenide, $\mathrm{Cu}_{2-}$ $\mathrm{SnSe}_{3}$, belonging to the $\mathrm{I}_{2} \mathrm{IVVI}_{3}$ family, is a $p$-type TE material having a narrow direct bandgap of $\sim 0.84$ $\mathrm{eV}$ [13]. $\mathrm{Cu}_{2} \mathrm{SnSe}_{3}$ obeys the phonon-glass-electroncrystal (PGEC) concept, which is usually exhibited by the widely investigated caged compounds like clathrates and skutterudites [1, 12-17]. This concept was proposed by Slack, which states that to achieve high TE performance in a material, the glass-like thermal conductivity and crystal-like electron transport must co-exist.

$\mathrm{Cu}_{2} \mathrm{SnSe}_{3}$ has been investigated from theoretical as well as experimental aspects for its peculiar transport properties $[15,16]$. In $\mathrm{Cu}_{2} \mathrm{SnSe}_{3}$, the highly distorted crystal structure allows achieving a very low lattice thermal conductivity. The $\mathrm{Cu}-\mathrm{Se}$ bond in $\mathrm{Cu}_{2} \mathrm{SnSe}_{3}$ is known to form an electrically conductive framework; thus, it dominates the $p$-type electrical transport of $\mathrm{Cu}_{2} \mathrm{SnSe}_{3}$ with little contribution from $\mathrm{Sn}$. Shi et al. suggested that the optimization of electrical properties can be achieved by doping different cations at the Sn site with a lower valence electron number than $\mathrm{Sn}$ [16]. Besides, substitution could also cause mass fluctuations that could lead to a stronger phonon scattering, and in turn, lower thermal conductivity. A record $Z T$ of 1.14 reported by Shi et al. [16] in $\mathrm{Cu}_{2-}$ $\mathrm{Sn}_{0.9} \mathrm{In}_{0.1} \mathrm{Se}_{3}$ at $850 \mathrm{~K}$ is close to the values obtained for the state-of-the-art TE materials like skutterudites and commercial $\mathrm{Bi}_{2} \mathrm{Te}_{3}$-based compounds [17, 18]. A drop in thermal conductivity was observed by doping $\mathrm{Ga}$ at $\mathrm{Sn}$ site as a result of mass fluctuation. Doping with Ga also led to the enhancement in carrier concentration and electrical conductivity; thus, an improved $Z T$ value of 0.43 at $700 \mathrm{~K}$ was attained [19]. Zhang et al. investigated the effect of Fe doping and reported a high ZT of 1.1 at $\sim 820 \mathrm{~K}$ for $x=0.05$ and 0.1 as a result of enhanced power factor in the $\mathrm{Cu}_{2} \mathrm{In}_{1-x} \mathrm{Fe}_{x} \mathrm{Se}_{3}$ system [20]. Doping with $\mathrm{Mn}, \mathrm{Pb}$, and $\mathrm{Sb}$ has also resulted in the enhancement of $\mathrm{ZT}$ [21-23]. Li et al. synthesized ( $\mathrm{Ag}, \mathrm{In}$ )-co-doped $\mathrm{Cu}_{2-}$ $\mathrm{SnSe}_{3}$ and a maximum $\mathrm{ZT}$ of 1.42 in $\mathrm{Cu}_{1.85} \mathrm{Ag}_{0.15-}$ $\mathrm{Sn}_{0.9} \mathrm{In}_{0.1} \mathrm{Se}_{3}$ at $823 \mathrm{~K}$ was achieved [24]. The stoichiometric tuning of $\mathrm{Cu}$ in $\mathrm{Cu}_{2} \mathrm{SnSe}_{3}$ has also been reported as an effective approach to attain a higher $Z T$ value [25].

In this study, the effect of doping on the TE transport properties in the temperature range $10-400 \mathrm{~K}$ by employing In as a dopant at the Sn site of $\mathrm{Cu}_{2} \mathrm{SnSe}_{3}$ has been studied. Generally, grain boundaries and point defects are the major scattering factors for electrons and phonons at low temperatures, while electron-phonon and phonon-phonon scatterings become dominant at higher temperatures. Therefore, the electrical and thermal transport measurements can provide valuable information regarding the various scattering mechanisms. Besides, the detailed investigation of the TE properties of $\mathrm{Cu}_{2} \mathrm{In}_{1-}$ ${ }_{x} \mathrm{Sn}_{x} \mathrm{Se}_{3}(x=0,0.05,0.10,0.15,0.20,0.25)$ revealed a significant increase in the power factor $\left(P F=S^{2} \sigma\right)$ together with a simultaneous decrease in thermal conductivity. This led to an enhancement in $\mathrm{ZT}$ by six times as compared to the pristine $\mathrm{Cu}_{2} \mathrm{SnSe}_{3}$.

\section{Experimental}

$\mathrm{Cu}_{2} \mathrm{In}_{1-x} \mathrm{Sn}_{x} \mathrm{Se}_{3}(x=0,0.05,0.10,0.15,0.20,0.25)$ samples were synthesized by the conventional solidstate reaction route [26-29] followed by furnace sintering. Commercial high-purity powders of $\mathrm{Cu}$ (99.7\%, Loba Chemie), Sn, In, and Se (99.999\%, Alfa Aesar) taken in the desired stoichiometric ratio were ground well and sealed in evacuated $\left(10^{-3}\right.$ Torr $)$ quartz tubes. These were then sintered in a muffle furnace at $500{ }^{\circ} \mathrm{C}$ for $72 \mathrm{~h}$ and allowed to cool naturally to room temperature.

The crystallinity and phase structure of the samples were analyzed by the X-ray diffraction (XRD) using Rigaku Miniflex equipped with 
monochromatic $\mathrm{Cu}-\mathrm{K} \alpha$ radiation. The binding energies of the constituent elements of $\mathrm{Cu}_{2} \mathrm{In}_{1-x} \mathrm{Sn}_{x} \mathrm{Se}_{3}$ were investigated using $\mathrm{X}$-ray photoelectron spectroscopy (XPS) of Thermo Scientific equipped with monochromated X-ray source of $\mathrm{Al} \mathrm{K \alpha}$. The microstructure of finely polished surfaces of the assynthesized samples was examined by field emission scanning electron microscopy (FESEM) using a JEOL JSM-7000F equipped with energy-dispersive X-ray spectroscopy (EDS; INCA, Oxford Instruments) which was utilized for the elemental analysis.

The measurement of electrical resistivity with respect to temperature (10-400 K) for the as-prepared samples was performed by employing the standard four-probe technique in closed-cycle refrigeration (CCR). The temperature-dependent Seebeck coefficient and thermal conductivity were determined simultaneously by adopting a direct heat-pulse technique. Further details of the measurement techniques are given elsewhere [30]. The carrier concentration and mobility of the samples were determined by employing the van der Pauw method using Hall Effect Measurement System (HMS-5500) with a magnetic field of $\pm 0.5 \mathrm{~T}$ at room temperature.

\section{Results and discussion}

\subsection{Structural and compositional characterization}

The room-temperature XRD patterns of pristine and In-doped $\mathrm{Cu}_{2} \mathrm{SnSe}_{3}$ samples are shown in Fig. 1a. It is observed that all the samples have maintained the cubic structure with space group $F \overline{4} 3 m$, consistent with earlier reports [20-23]. It is noticed that traces of SnSe as impurity phase can be seen in samples with $x=0,0.05,0.10$, and 0.15 in $\mathrm{Cu}_{2} \mathrm{In}_{1-x} \mathrm{Sn}_{x} \mathrm{Se}_{3}$. The peaks for the In-doped samples show a slight shift towards the lower $2 \theta$ side. This can be clearly observed in the enlarged (111) peaks, as shown in the inset of Fig. 1a, indicative of enlargement of the unit cell with In doping. Further, the quantitative analysis of the experimental XRD data was carried out by employing the Rietveld refinement technique using the FULLPROF program [31, 32], and the fitting parameters are given in Table 1 . The observed $\left(Y_{\mathrm{obs}}\right)$ and calculated $\left(Y_{\text {cal }}\right)$ patterns, along with their difference, are depicted in Fig. $1 b$, and the simulated patterns fit well with the obtained XRD data. The deduced lattice parameter $a(=b=c)$ shows a linear increase with an increase in $x$ (Fig. 1c), which is in agreement with the Vegard's law. This is due to the larger ionic radius of In than $\mathrm{Sn}$, which leads to the expansion of the crystal lattice. Similar results were also reported for In doping on the $\mathrm{Sn}$ site of $\mathrm{Cu}_{2} \mathrm{SnS}_{3}$ [33].

Figure $2 \mathrm{a}$ presents the micrographs of FESEM performed on the polished surfaces of $\mathrm{Cu}_{2} \mathrm{In}_{1-x} \mathrm{Sn}_{x-}$ $\mathrm{Se}_{3}$ for $x=0$ and 0.25 samples. Minimal pores or cracks are visible in the samples' surface morphology, which indicates the compactness and homogeneity of the samples. The morphology of all the synthesized samples is similar with the size of the clusters ranging from several hundred nanometers to several micrometers, and these clusters do not exhibit any obvious variation with increasing in In content. The results of the EDS mapping carried out on $x=0.25$ sample given in Fig. $2 \mathrm{~b}$ suggests that all the elements $(\mathrm{Cu}, \mathrm{Sn}, \mathrm{In}$, and Se) present are evenly distributed with no detectable phase segregation. The elemental analysis suggests that the influence of the secondary phase or uneven elemental distribution on the thermoelectric properties of our studied samples is negligible.

The valence states of $\mathrm{Cu}, \mathrm{Sn}, \mathrm{In}$, and $\mathrm{Se}$ in $\mathrm{Cu}_{2} \mathrm{In}_{1-}$ ${ }_{x} \mathrm{Sn}_{x} \mathrm{Se}_{3}(x=0,0.05,0.10,0.15,0.20,0.25)$ are confirmed by employing X-ray photoelectron spectroscopy (XPS), and the resulting survey and corelevel spectra of the respective elements are presented in Fig. 3a. The absence of any other peak except $\mathrm{Cu}$, $\mathrm{Sn}, \mathrm{Se}, \mathrm{In}, \mathrm{O}$, and $\mathrm{C}$ in Fig. $3 \mathrm{a}$ indicates the high purity of the prepared samples. Figure $3 \mathrm{~b}$ shows the doublet peaks located at binding energies $\sim 932.1$ and $\sim 951.9 \mathrm{eV}$ with a standard separation of $19.8 \mathrm{eV}$. It is revealed from the literature that these peaks correspond to $\mathrm{Cu} 2 p_{3 / 2}$ and $2 p_{1 / 2}$, respectively, confirming the presence of $\mathrm{Cu}^{+}$in the samples [34, 35]. No peak is seen around $942 \mathrm{eV}$, and this excludes the presence of $\mathrm{Cu}^{2+}$ in the samples. For $\mathrm{Sn}$ $3 d$ spectrum in Fig. $3 \mathrm{c}$, the two peaks at $486.3\left(\mathrm{Sn} 3 d_{5}\right.$, $\left.{ }_{2}\right)$ and $494.7 \mathrm{eV}\left(\mathrm{Sn} 3 d_{3 / 2}\right)$ refer to the existence of $\mathrm{Sn}^{4+}$. Figure $3 \mathrm{~d}$ exhibits the core-level spectrum of In in the doped samples, where the doublet states at binding energies, $444.6 \mathrm{eV}$ and $452.2 \mathrm{eV}$ correspond to In $3 d_{5 / 2}$ and $3 d_{3 / 2}$ of $\operatorname{In}^{3+}$, respectively [30,31]. The asymmetric peak of Se $3 d$ at $\sim 54.7 \mathrm{eV}$ signifies Se (Fig. 3e) with the valence state of -2 . These results obtained agree well with those available in the literature [34-37]. 

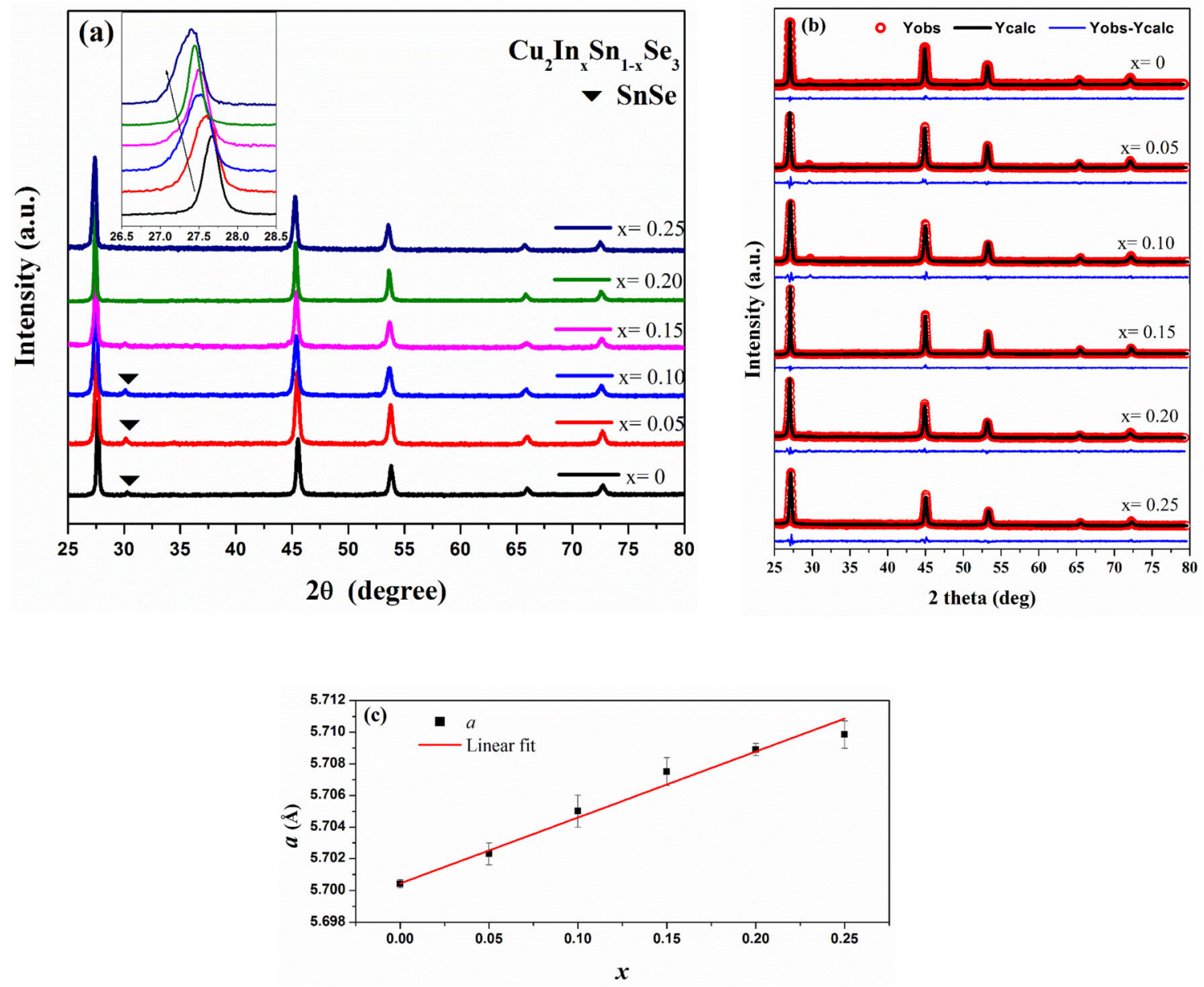

Fig. 1 a Room-temperature XRD pattern, b Rietveld refinement of $\mathrm{Cu}_{2} \operatorname{In}_{1-x} \mathrm{Sn}_{x} \mathrm{Se}_{3}(x=0,0.05,0.10,0.15,0.20,0.25)$ samples, and c linearly varying lattice parameter as a function of $x$

Table 1 Crystal structure parameters of $\mathrm{Cu}_{2} \mathrm{In}_{1-x} \mathrm{Sn}_{x} \mathrm{Se}_{3}$ $(x=0,0.05,0.10,0.15,0.20$, $0.25)$ samples obtained from refinement of XRD

\begin{tabular}{lllll}
\hline$x$ in $\mathrm{Cu}_{2} \mathrm{In}_{1-\mathrm{x}} \mathrm{Sn}_{\mathrm{x}} \mathrm{Se}_{3}$ & Crystal structure & Lattice parameter $a(\AA)$ & $\chi^{2}$ & $R_{w p} \%$ \\
\hline 0 & Cubic $F \overline{4} 3 m$ & 5.6995 & 1.17 & 11.0 \\
0.05 & Cubic $F \overline{4} 3 m$ & 5.7004 & 1.59 & 11.2 \\
0.10 & Cubic $F \overline{4} 3 m$ & 5.7050 & 1.50 & 11.6 \\
0.15 & Cubic $F \overline{4} 3 m$ & 5.7075 & 1.39 & 11.8 \\
0.20 & Cubic $F \overline{4} 3 m$ & 5.7081 & 1.40 & 11.2 \\
0.25 & Cubic $F \overline{4} 3 m$ & 5.7093 & 1.49 & 11.5 \\
\hline
\end{tabular}

\subsection{Thermoelectric transport properties}

The variation of electrical resistivity with temperature $\rho(T)$ in the range of $10-400 \mathrm{~K}$ for pure and Indoped $\mathrm{Cu}_{2} \mathrm{SnSe}_{3}$ samples is illustrated in Fig. 4a. Several features in $\rho(T)$ are observed with an increase in In concentration. Firstly, the samples with $x=0$, 0.05 , and 0.10 show a semiconducting behavior where the electrical resistivity decreases with an increase in temperature $(d \rho / d T<0)$. With further increase in In content, i.e., for $x=0.15,0.20$, and 0.25 , the nature of $\rho(T)$ changes to heavily doped semiconductor or metallic behavior $(d \rho / d T>0)$. Secondly, the overall value of $\rho(T)$ decreases with an increase in $x$, where the $\rho(T)$ of $x=0.25$ is nearly two orders of magnitude smaller than that of the pristine sample. The room-temperature $\rho(T)$ reduces from $0.0765 \Omega \mathrm{cm}$ for $x=0$ to $0.0343,0.0196,0.0024,0.0018$, 
Fig. 2 a FESEM micrographs of $x=0$ and 0.25 of $\mathrm{Cu}_{2} \mathrm{In}_{1-x} \mathrm{Sn}_{x} \mathrm{Se}_{3}$ samples, b EDS mapping of $\mathrm{Cu}_{2} \mathrm{In}_{0.25} \mathrm{Sn}_{0.75} \mathrm{Se}_{3}$ for the respective elements (a)
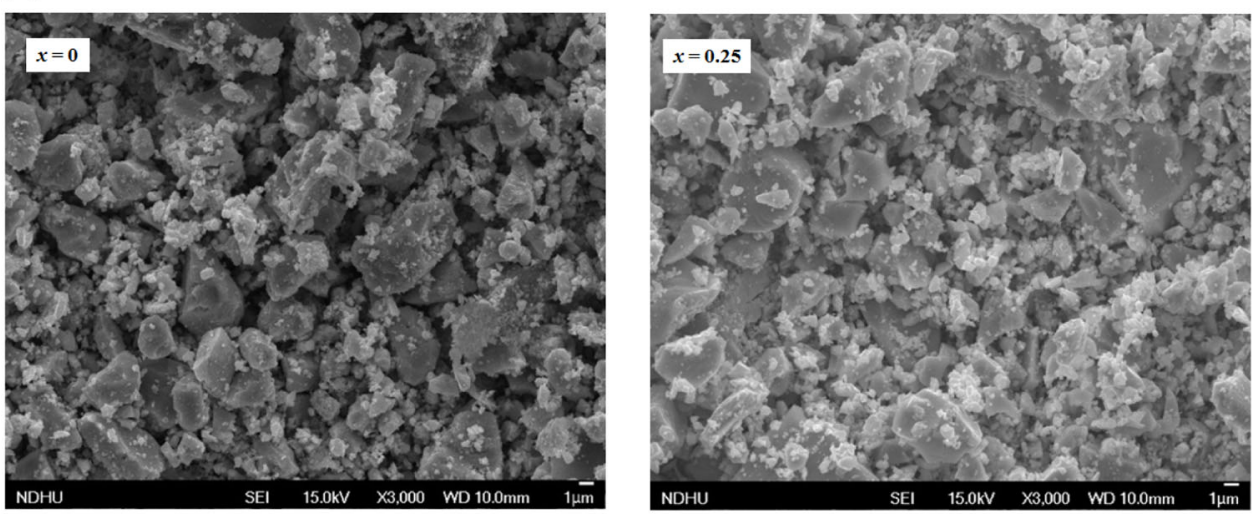

(b)

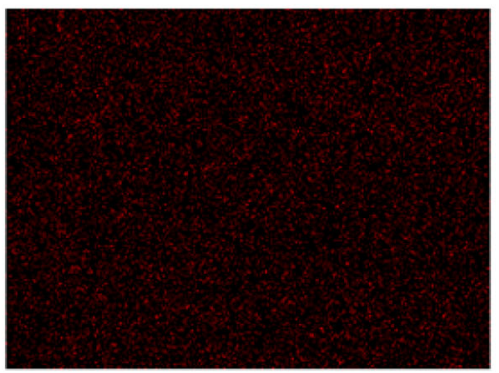

Cu Ka1

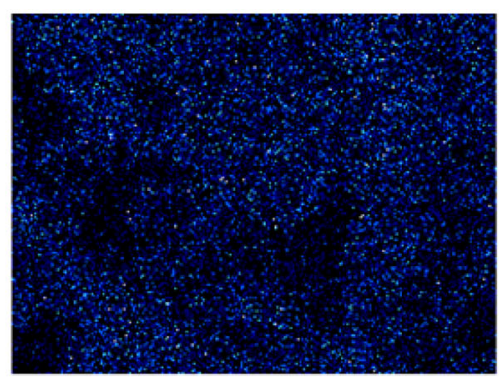

Sn La1

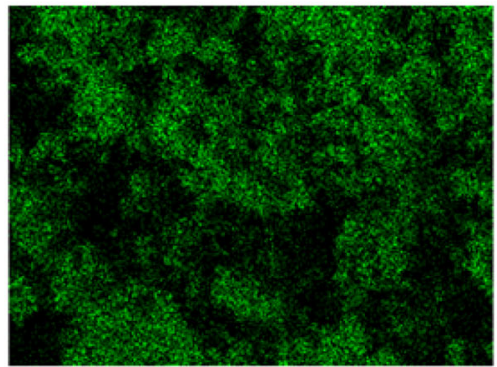

Se La1_2

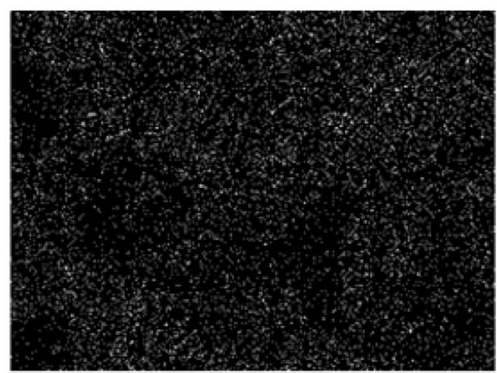

In La1 and $0.0010 \Omega \mathrm{cm}$ for samples with $x=0.05,0.10,0.15$, 0.20 , and 0.25 , respectively. More holes are expected to be created with an increase in In concentration in the samples that act as an electron acceptor due to the substitution of +3 valent In on the +4 valent $S n$ site. This results in an enhancement in hole concentration, which in turn leads to the drop in electrical resistivity. Such a decreasing trend in electrical resistivity with an increase in dopant concentration was also reported in the literature [19-21, 38]. The $\rho(T)$ of heavily doped samples $(x=0.15,0.20$, and 0.25$)$ shows a $T \sim 1.5$ dependence (inset of Fig. $4 a$ ), indicating the dominance of acoustic phonon scattering in the highly degenerate state.
The observations of electrical resistivity are further supported by room-temperature Hall measurements, and the results are presented in Table 2. It can be observed that the carrier concentration $p$ is effectively enhanced with In doping, reflecting the acceptor role of the In atoms in the $\mathrm{Cu}_{2} \mathrm{In}_{1-x} \mathrm{Sn}_{x} \mathrm{Se}_{3}$ system. The overall decrease in carrier mobility in In-doped samples as compared to the undoped can be attributed to the extra ionized impurity scattering and enhanced carrier scattering. A similar trend in Hall measurements is also observed for $\mathrm{Ga}$ and $\mathrm{Zn}$-doped $\mathrm{Cu}_{2} \mathrm{SnSe}_{3}[19,38]$.

The plot of Seebeck coefficient $S(T)$ vs. temperature for the $\mathrm{Cu}_{2} \mathrm{In}_{1-x} \mathrm{Sn}_{x} \mathrm{Se}_{3}$ series is illustrated in Fig. $4 \mathrm{~b}$. It can be observed that all studied samples exhibit 

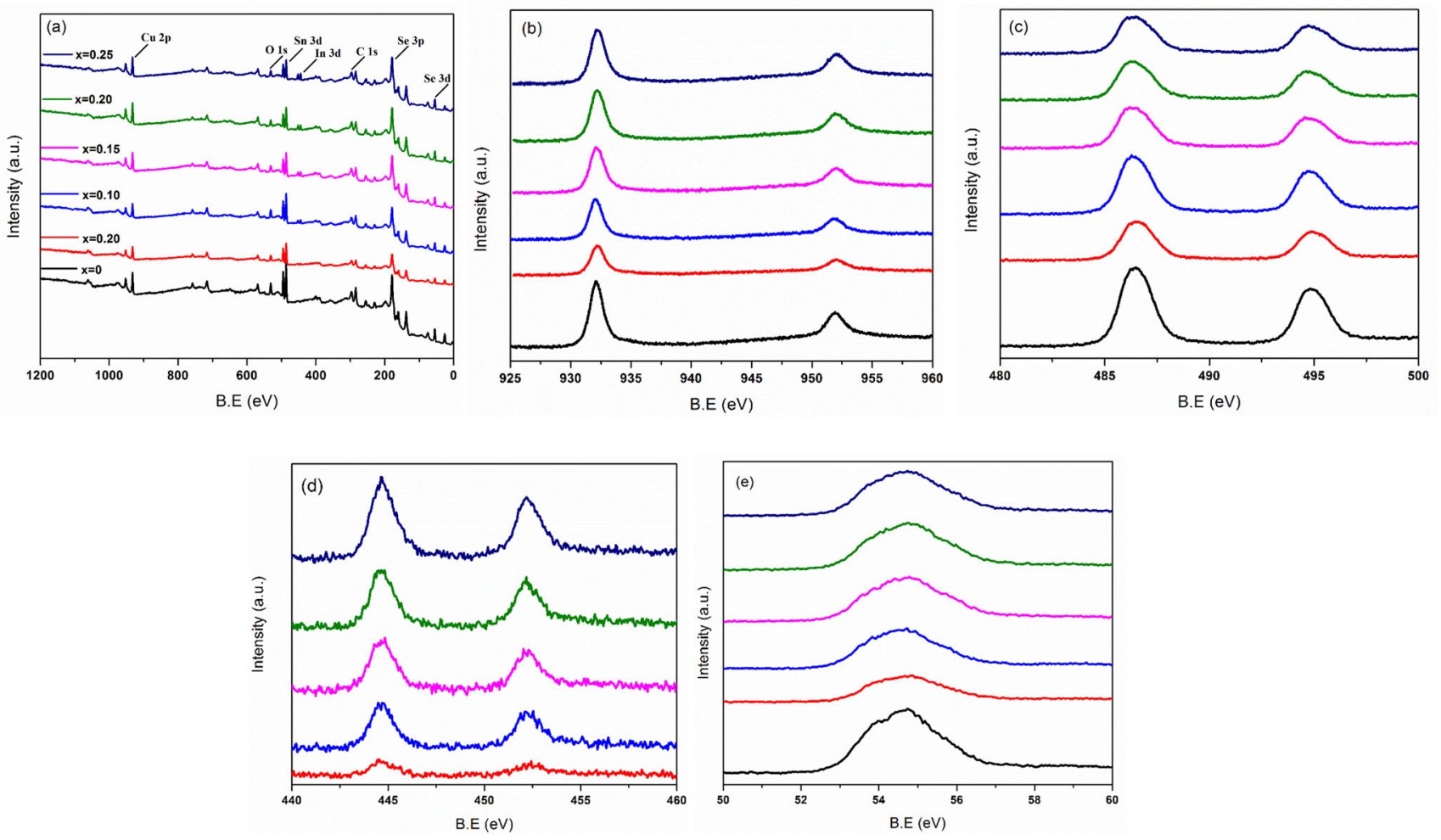

Fig. 3 a XPS survey spectrum of $\mathrm{Cu}_{2} \mathrm{In}_{1-x} \mathrm{Sn}_{x} \mathrm{Se}_{3}(x=0,0.05,0.10,0.15,0.20,0.25)$ samples. High-resolution spectra of $\mathbf{b} \mathrm{Cu} 2 p, \mathbf{c} \mathrm{Sn}$ $3 d$, d In $3 d$ and e Se $3 d$ in $\mathrm{Cu}_{2} \operatorname{In}_{1-x} \mathrm{Sn}_{x} \mathrm{Se}_{3}(x=0,0.05,0.10,0.15,0.20,0.25)$ samples
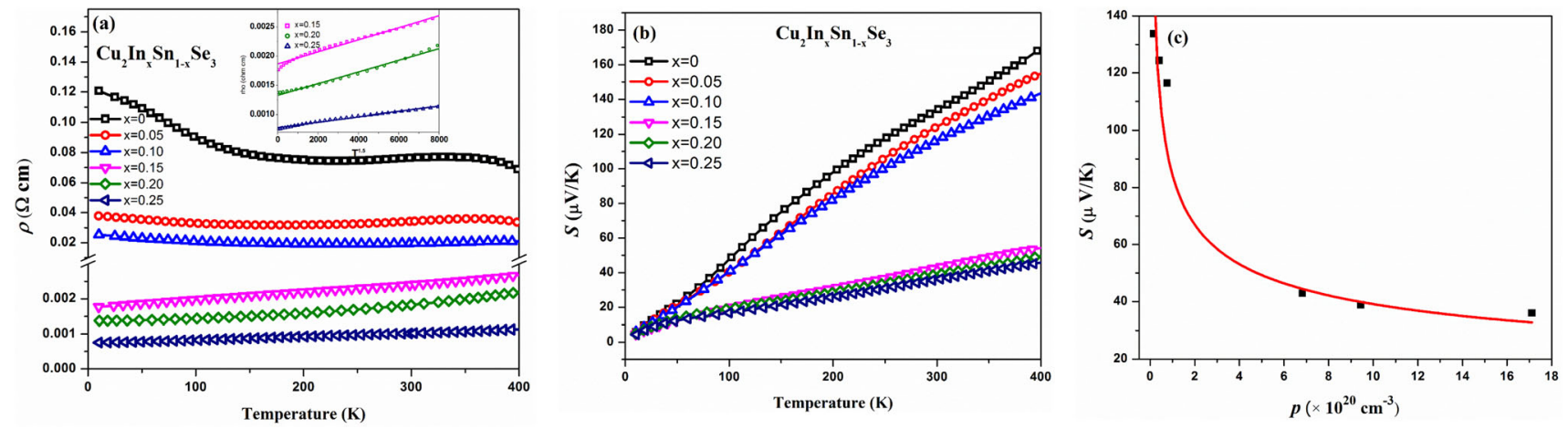

Fig. 4 a Temperature-dependent resistivity with insets showing $\mathrm{T}^{1.5}$ dependency, $\mathbf{b}$ Seebeck coefficient of $\mathrm{Cu}_{2} \mathrm{In}_{1-x} \mathrm{Sn}_{x} \mathrm{Se}_{3}(x=0,0.05$, $0.10,0.15,0.20,0.25)$ samples, and $\mathbf{c} S \sim p^{-1 / 3}$ dependence of linear band approximation model

positive values of $S(T)$ for the whole temperature range suggesting the $p$-type thermoelectric conduction for $\mathrm{Cu}_{2} \mathrm{In}_{1-x} \mathrm{Sn}_{x} \mathrm{Se}_{3}$, in agreement with the Hall measurements. Besides, it is observed that $S(T)$ increases with increasing temperature. The almost linear increase of $S(T)$ for doped samples with temperature indicates the heavily doped semiconducting behavior and follows the trend as reported for doped $\mathrm{Cu}_{2} \mathrm{SnSe}_{3}$ [19-21]. However, $S(T)$ decreases significantly with the increase in $x$, and this is attributed to the inverse proportionality of $S(T)$ with respect to $p$. For example, the room-temperature value of $S(T)$ decreases from $\sim 134 \mu \mathrm{V} \mathrm{K}{ }^{-1}$ for the pristine sample to $\sim 36 \mu \mathrm{V} \mathrm{K}^{-1}$ for the $x=0.25$ sample. For heavily doped semiconductors, this inverse relationship can be described by Mott's equation with an assumption of a single parabolic band (SPB) model and is given as $[23,38]$ : 
Table 2 Carrier concentration $p$, hall mobility $\mu$, density of states (DOS) effective mass $\mathrm{m}^{*} / \mathrm{m}_{e}$, and Fermi energy $E_{F}$ of $\mathrm{Cu}_{2} \operatorname{In}_{1-x} \mathrm{Sn}_{x} \mathrm{Se}_{3}(x=0,0.05,0.10,0.15,0.20,0.25)$

\begin{tabular}{lllll}
\hline$x$ & $p\left(10^{20} \mathrm{~cm}^{-3}\right)$ & $\mu\left(\mathrm{cm}^{2} \mathrm{~V}^{-1} \mathrm{~s}^{-1}\right)$ & $m^{*} / m_{e}$ & $E_{f}(\mathrm{eV})$ \\
\hline 0 & 0.160 & 5.106 & 0.423 & -0.055 \\
0.05 & 0.405 & 4.550 & 0.724 & -0.059 \\
0.10 & 0.753 & 4.235 & 1.034 & -0.063 \\
0.15 & 6.821 & 3.809 & 1.656 & -0.171 \\
0.20 & 9.432 & 3.626 & 1.862 & -0.189 \\
0.25 & 17.112 & 3.577 & 2.567 & -0.203 \\
\hline
\end{tabular}

$S=\frac{8 k_{B}^{2} \pi^{2}}{3 e h^{2}} m^{*}\left(\frac{\pi}{3 n}\right)^{2 / 3} T$

where $e$ is the elementary charge, $k_{B}$ is the Boltzmann constant, $h$ is the Planck's constant, and $m^{*}$ is the effective mass of the electron. The features of thermoelectric conduction are elucidated by determining the effective mass $m^{*}$ and Fermi energy of the samples at room temperature from the experimental $S$ and $p$ values by using Eqs. (1) and (2), respectively $[23,39]$.

$E_{F}=\frac{-\pi^{2} k_{B}^{2} T}{3 e S}$

From the results presented in Table 2, it is noticed that $m^{*}$ shows a clear increase with an increase in dopant concentration, which is in accordance with the decrease in hole mobility at elevated carrier concentrations. The negative values of $E_{F}$ suggest that the Fermi energy is below the valence band, and it shifts into deeper levels with the increase in $x$. In addition, the electrical properties of $\mathrm{Cu}_{2} \mathrm{In}_{1-x} \mathrm{Sn}_{x} \mathrm{Se}_{3}$ are also examined by exploring the dependence of $S$ vs. $p$ at $300 \mathrm{~K}$ (Fig. $4 \mathrm{c}$ ). It can be observed that the linear band dispersion model proposed by Ioffe [40] gives a good fit for the experimental data, suggesting that all the samples follow the relation of $S \sim p^{-1 / 3}$. This indicates that In doping at the Sn site has weak influences on the band structure; instead, the electrical transport in these samples is predominantly governed by carrier concentration. This model was also applied for $\mathrm{Cu}_{2} \mathrm{Ga}_{1-x} \mathrm{Sn}_{x} \mathrm{Se}_{3}$ [19], filled skutterudites, and clathrates [41, 42].

The temperature dependence of total thermal conductivity $\kappa(T)$ for the pristine and doped samples is depicted in Fig. 5a. The thermal conductivity of all studied samples exhibits a similar trend with temperature, which is the characteristic behavior of crystalline solids [43]. It is seen that $\kappa(T)$ increases sharply with increasing temperature, followed by a distinct maximum (Umklapp peak) at $\sim 35 \mathrm{~K}$. Above this temperature, $\kappa(T)$ gradually decreases. This behavior is attributed to the grain boundary scattering at low temperatures and the Umklapp process at high temperatures. Further inspection of the thermal transport behavior is carried out by evaluating $\kappa_{e}$ from the Wiedemann-Franz law: $\kappa_{e}(-$ $T)=L_{0} T / \rho$; where $L_{0}$ is the Lorenz number and $\rho$ is the electrical resistivity at temperature $T$. Kim et al. have proposed a simplified assessment of $L_{0}$ for
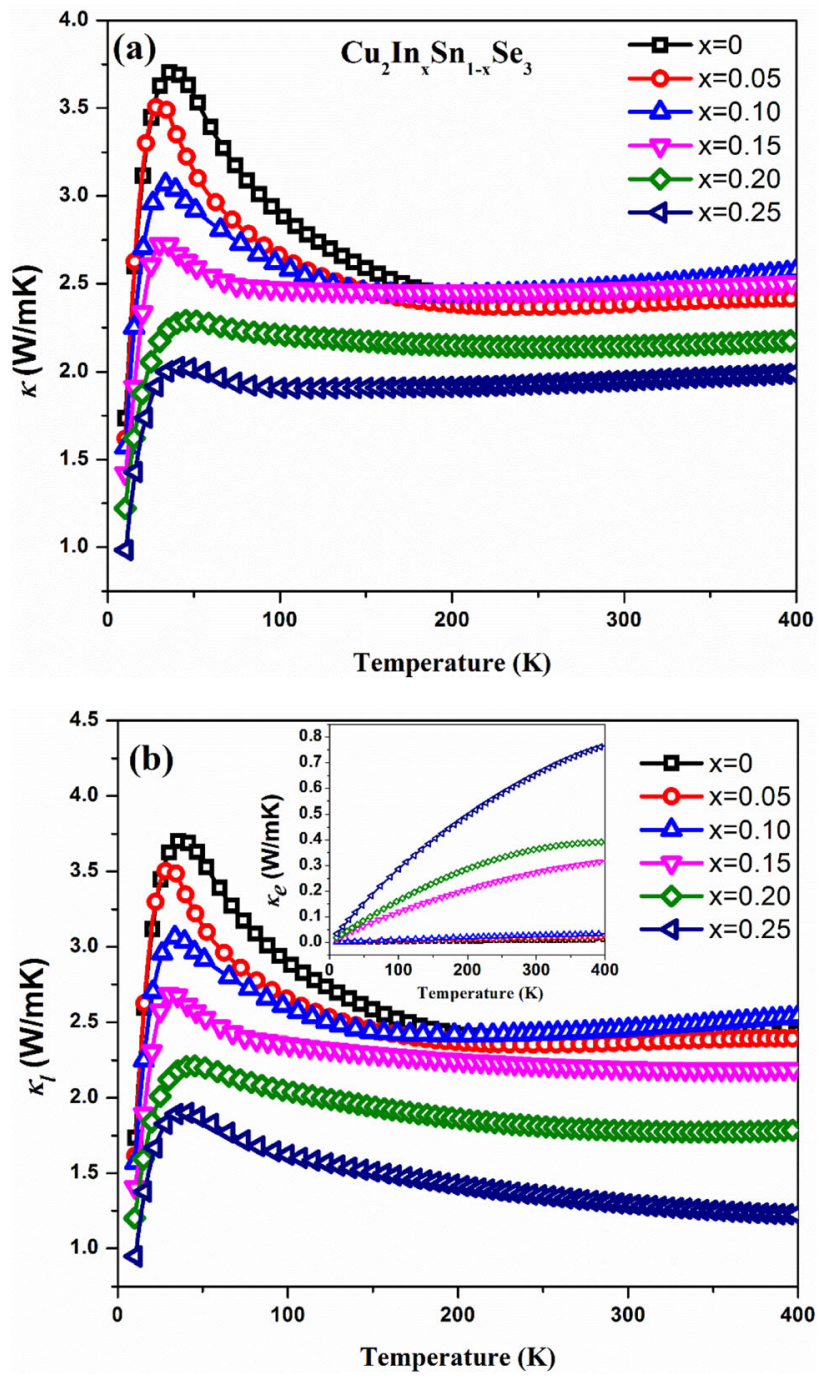

Fig. 5 a Temperature-dependent total thermal conductivity and b lattice thermal conductivity with inset showing the electrical contribution for $\mathrm{Cu}_{2} \mathrm{In}_{1-x} \mathrm{Sn}_{x} \mathrm{Se}_{3}(x=0,0.05,0.10,0.15,0.20$, $0.25)$ samples 
semiconductors from $S(T)$ data which is expressed in Eq. (3) [9],

$L_{0}=1.5+\exp \left[\frac{-|S|}{116}\right]$

Here $L_{0}$ is in the units of $10^{-8} \mathrm{~W} \Omega \mathrm{K}^{-2}$, and $S$ is in $\mu \mathrm{V} \mathrm{K}^{-1}$. The electronic contribution of thermal conductivity $\kappa_{e}(T)$ is obtained using Eq. (3) and is shown in the inset of Fig. 5b. The value of $\kappa_{e}(T)$ for the pristine sample is very low due to its high electrical resistivity; however, $\kappa_{e}(T)$ increases with increasing In concentration. Since the measured $\kappa(T)$ is the sum of electronic and lattice contributions, the lattice thermal conductivity $\kappa_{L}(T)$ can be obtained from $\kappa_{L}(T)=\kappa(T)-\kappa_{e}(T)$. Figure $5 \mathrm{~b}$ demonstrates the temperature dependence of $\kappa_{L}$, and it is much larger than $\kappa_{e}$, indicating that the lattice contribution dominates heat conduction in the $\mathrm{Cu}_{2} \mathrm{In}_{1-x} \mathrm{Sn}_{x} \mathrm{Se}_{3}$ system. In addition, $\kappa_{L}(T)$ decreases with increasing temperature following roughly a $T^{-1}$ law at high temperatures. This suggests that the phonon-phonon Umklapp process is predominant, and no obvious bipolar diffusion effect is involved in the total thermal conductivity of $\mathrm{Cu}_{2} \mathrm{In}_{1-x} \mathrm{Sn}_{x} \mathrm{Se}_{3}$. Besides, it is noticed that the lattice thermal conductivity decreases systematically with increasing In content. The mass fluctuations induced by the In doping could cause lattice distortions and create point defects, which would lead to enhanced phonon scattering and, in turn, lower the thermal conductivity.

\subsection{Power factor and figure of merit}

An important quantity for optimization of thermoelectric materials, namely the power factor $(P F)$, is expressed as $P F=S^{2} / \rho$. The temperature dependence of $P F$ is obtained by using the experimental results and is shown in Fig. 6a. With increasing In content, an enhancement in the value of $P F$ for the $\mathrm{Cu}_{2} \mathrm{In}_{1-x} \mathrm{Sn}_{x} \mathrm{Se}_{3}$ series is achieved, mainly due to the significant reduction of the electrical resistivity. The highest $P F$ of $\sim 185 \mu \mathrm{W} \mathrm{m}{ }^{-1} \mathrm{~K}^{-2}$ for the $x=0.25$ sample is attained, which is more than four times larger than that of the pristine sample $\left(\sim 41 \mu \mathrm{W} \mathrm{m}{ }^{-1} \mathrm{~K}^{-2}\right)$ at $400 \mathrm{~K}$.

Based on the measured electrical and thermal transport parameters, the dimensionless figure of merit was calculated as $Z T=\frac{S^{2}}{\rho \kappa} T$. The $Z T$ as a function of temperature for $\mathrm{Cu}_{2} \mathrm{In}_{1-x} \mathrm{Sn}_{x} \mathrm{Se}_{3}(x=0$,
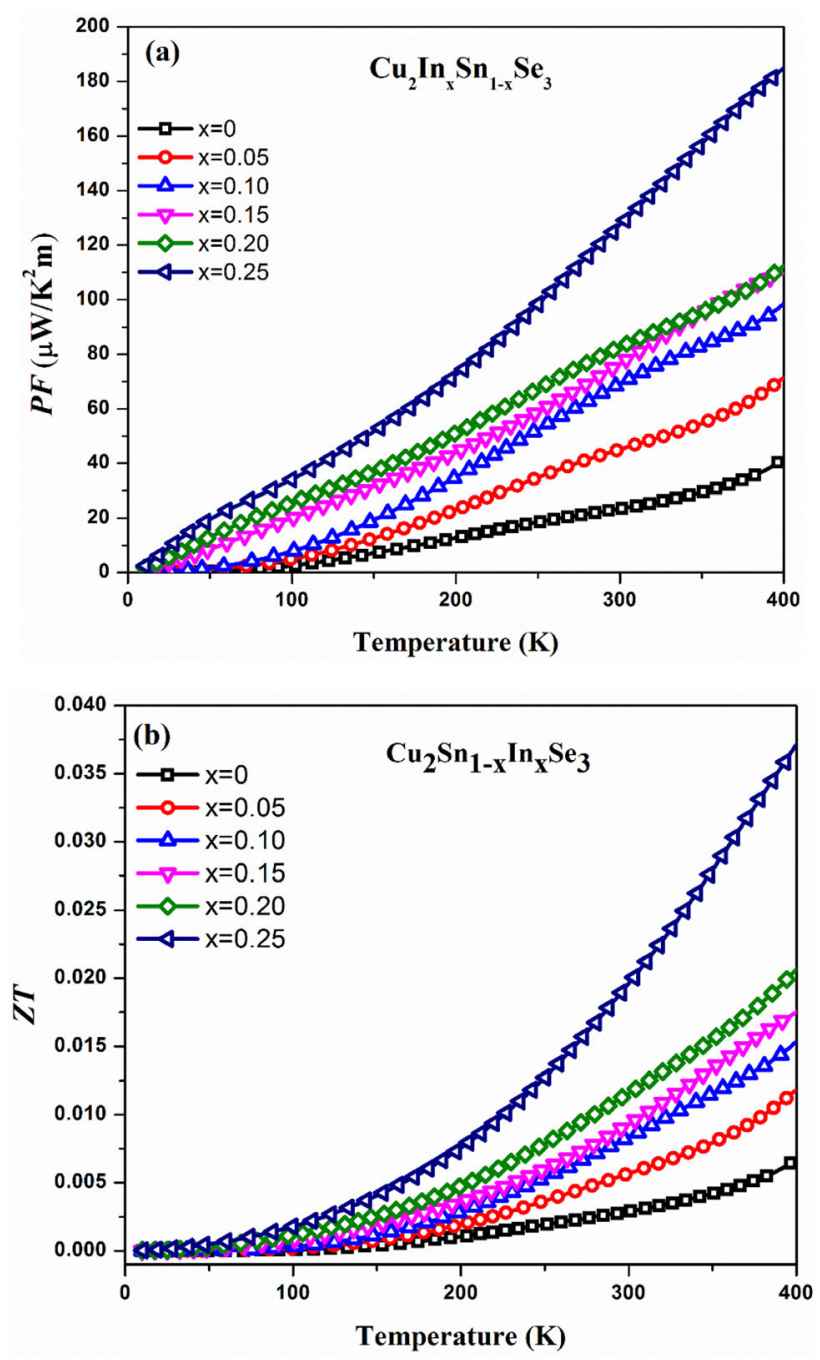

Fig. 6 a Temperature dependence of power factor and $\mathbf{b} Z T$ of $\mathrm{Cu}_{2} \mathrm{In}_{1-x} \mathrm{Sn}_{x} \mathrm{Se}_{3}(x=0,0.05,0.10,0.15,0.20,0.25)$ samples

$0.05,0.10,0.15,0.20,0.25)$ is depicted in Fig. $6 \mathrm{~b}$. The enhancement in $P F$, along with the reduction in $\kappa(T)$, leads to an increase in ZT with the increase in $x$. More than a factor of six enhancement in $Z T$ is achieved in comparison with the pristine sample, with the maximum $Z T \sim 0.04$ for the $x=0.25$ sample at $400 \mathrm{~K}$. Moreover, further improvement in $Z T$ is expected at higher temperatures ( $>400 \mathrm{~K}$ ), which warrants further investigation of high-temperature TE measurements for these samples. 


\section{Conclusion}

In this communication, In is selected as a dopant at the Sn site of $\mathrm{Cu}_{2} \mathrm{SnSe}_{3}$ and its effect on the structural and thermoelectric properties is meticulously investigated in the temperature regime of $10-400 \mathrm{~K} . \mathrm{Cu}_{2-}$ $\mathrm{In}_{1-x} \mathrm{Sn}_{x} \mathrm{Se}_{3}(x=0,0.05,0.10,0.15,0.20,0.25)$ samples prepared by the solid-state reaction sintering technique adopt a cubic structure which is analyzed by $\mathrm{X}$-ray diffraction studies. The quantitative investigation of the XRD patterns by the Rietveld refinement technique reveals the increase in lattice parameter $a$ with increasing In concentration, indicating the successful substitution of Sn by In. The SEM-EDS measurements were performed to study the morphology of the samples. The SEM-EDS images reveal the homogeneous distribution of the constituent elements in $\mathrm{Cu}_{2} \mathrm{In}_{1-x} \mathrm{Sn}_{x} \mathrm{Se}_{3}$ with little porosity. The valence states of the elements of all the prepared samples are confirmed by XPS, and the results agree well with the literature. From the analysis of the measured Seebeck coefficient of the $\mathrm{Cu}_{2} \mathrm{In}_{1-x} \mathrm{Sn}_{x} \mathrm{Se}_{3}$ series, we conclude that In acts as an acceptor dopant and has a strong influence on the thermoelectric properties of $\mathrm{Cu}_{2} \mathrm{SnSe}_{3}$. As the In content increases, both the electrical resistivity and Seebeck coefficient decrease. This is due to the increase in hole concentration, as In has one less valence electron than that of $\mathrm{Sn}$. The enhancement in carrier concentration is confirmed by Hall measurements at room temperature. The positive values of $S(T)$ and the Hall coefficient indicate the $p$-type conduction in all the studied samples. The increase in electrical conductivity with In doping leads to a significant increase in PF. The mass fluctuations introduced by In doping increases the phonon scattering, which in turn reduces the thermal conductivity. The maximum ZT obtained for the $x=0.25$ sample is about six times higher than that of the pristine sample. Moreover, the $Z T$ of the $\mathrm{Cu}_{2-}$ $\mathrm{In}_{1-x} \mathrm{Sn}_{x} \mathrm{Se}_{3}$ system might be further improved by a more effective reduction in $\kappa(T)$ by using a nanostructuring approach.

\section{Acknowledgements}

This research was financially supported by the Council of Scientific and Industrial Research Grant (sanction no.: 03(1409)/17/E MR-II), Department of Science and Technology, India, DST-FIST Grant (SR/
FIST/PS-1/2017/8), and the Ministry of Science and Technology of Taiwan under Grant Nos. MOST-1062112-M-312 259-002-MY3 and MOST 107-2112-M-259004 (YKK).

\section{Funding}

Open access funding provided by Manipal Academy of Higher Education, Manipal.

Open Access This article is licensed under a Creative Commons Attribution 4.0 International License, which permits use, sharing, adaptation, distribution and reproduction in any medium or format, as long as you give appropriate credit to the original author(s) and the source, provide a link to the Creative Commons licence, and indicate if changes were made. The images or other third party material in this article are included in the article's Creative Commons licence, unless indicated otherwise in a credit line to the material. If material is not included in the article's Creative Commons licence and your intended use is not permitted by statutory regulation or exceeds the permitted use, you will need to obtain permission directly from the copyright holder. To view a copy of this licence, visit http://creativecommons.org/licen ses/by/4.0/.

\section{References}

1. D.M. Rowe (ed.), CRC Handbook of Thermoelectrics (CRC Press, Boca Raton, 1995)

2. G.J. Snyder, E.S. Toberer, Nat. Mater. 7, 105 (2008)

3. X.F. Zheng, C.X. Liu, Y.Y. Yan, Q. Wang, Renew. Sustain. Energy Rev. 32, 486 (2014)

4. G.D. Mahan, Appl. Mater. 4, 104806 (2016)

5. C. Gayner, K.K. Kar, Prog. Mater. Sci. 83, 330 (2016)

6. G. Chen, M.S. Dresselhaus, G. Dresselhaus, J.-P. Fleurial, T. Caillat, Int. Mater. Rev. 48, 45 (2003)

7. W. Liu, J. Hu, S. Zhang, M. Deng, C. Han, Y. Liu, Mater. Today Phys. 1, 50 (2017)

8. Z.-G. Chen, G. Han, L. Yang, L. Cheng, J. Zou, Prog. Nat. Sci. Mater. Int. 22, 535 (2012)

9. W. Kim, J. Mater. Chem. C 3, 10336 (2015)

10. P. Qiu, X. Shi, L. Chen, Energy Storage Mater. 3, 85 (2016)

11. E.J. Skoug, J.D. Cain, D.T. Morelli, J. Electron. Mater. 41, 1232 (2012)

12. D.T. Morelli, E.J. Skoug, MRS Proc. 1166, 157 (2009) 
13. G. Marcano, C. Rincón, L.M. De Chalbaud, D.B. Bracho, G.S. Pérez, J. Appl. Phys. 90, 1847 (2001)

14. T.-R. Wei, Y. Qin, T. Deng, Q. Song, B. Jiang, R. Liu, P. Qiu, X. Shi, L. Chen, Sci. China Mater. 62, 8 (2019)

15. L. Xi, Y.B. Zhang, X.Y. Shi, J. Yang, X. Shi, L.D. Chen, W. Zhang, J. Yang, D.J. Singh, Phys. Rev. B 86, 155201 (2012)

16. X. Shi, L. Xi, J. Fan, W. Zhang, L. Chen, Chem. Mater. 22, 6029 (2010)

17. G.S. Nolas, D.T. Morelli, T.M. Tritt, Annu. Rev. Mater. Sci. 29, 89 (1999)

18. O. Yamashita, S. Tomiyoshi, K. Makita, J. Appl. Phys. 93, 368 (2003)

19. J. Fan, H. Liu, X. Shi, S. Bai, X. Shi, L. Chen, Acta Mater. 61, 4297 (2013)

20. J. Zhang, L.L. Huang, X.G. Zhu, Z.M. Wang, C.J. Song, H.X. Xin, D. Li, X.Y. Qin, Scr. Mater. 159, 46 (2019)

21. X. Lu, D.T. Morelli, J. Electron. Mater. 41, 1554 (2012)

22. S. Prasad, A. Rao, B. Gahtori, S. Bathula, A. Dhar, C.-C. Chang, Y.-K. Kuo, Phys. B 520, 7 (2017)

23. K.S. Prasad, A. Rao, N.S. Chauhan, R. Bhardwaj, A. Vishwakarma, K. Tyagi, Appl. Phys. A 124, 98 (2018)

24. Y. Li, G. Liu, T. Cao, L. Liu, J. Li, K. Chen, L. Li, Y. Han, M. Zhou, Adv. Funct. Mater. 26, 6025 (2016)

25. S. Prasad, A. Rao, B. Christopher, R. Bhardwaj, N.S. Chauhan, S.A. Malik, N. Van Nong, B.S. Nagaraja, R. Thomas, J. Alloys Compd. 748, 273 (2018)

26. K. Shan, Z.-Z. Yi, X.-T. Yin, D. Dastan, F. Altaf, H. Garmestani, F.M. Alamgir, Surf. Interfaces 21, 100762 (2020)

27. W. Hu, T. Li, X. Liu, D. Dastan, K. Ji, P. Zhao, J. Alloys Compd. 818, 152933 (2020)

28. K. Shan, Z.-Z. Yi, X.-T. Yin, D. Dastan, H. Garmestani, Dalt. Trans. 49, 8549 (2020)

29. K. Shan, Z.Z. Yi, X.T. Yin, D. Dastan, H. Garmestani, Dalt. Trans. 49, 6682 (2020)
30. Y.-K. Kuo, B. Ramachandran, C.-S. Lue, Front. Chem. 2, 1 (2014)

31. K. Shan, Z.Z. Yi, X.T. Yin, L. Cui, D. Dastan, H. Garmestani, F.M. Alamgir, J. Alloys Compd. 855, 157465 (2021)

32. K. Shan, Z.Z. Yi, X.T. Yin, D. Dastan, S. Dadkhah, B.T. Coates, H. Garmestani, Adv. Powder Technol. 31, 4657 (2020).

33. Q. Tan, W. Sun, Z. Li, J.-F. Li, J. Alloys Compd. 672, 558 (2016)

34. J.M. Song, Y. Liu, H.L. Niu, C.J. Mao, L.J. Cheng, S.Y. Zhang, Y.H. Shen, J. Alloys Compd. 581, 646 (2013)

35. S. Li, D. Pan, J. Cryst. Growth. 358, 38 (2012)

36. V.B. Ghanwat, S.S. Mali, C.S. Bagade, R.M. Mane, C.K. Hong, P.N. Bhosale, Energy Technol. 4, 835 (2016)

37. J. Lei, W. Guan, D. Zhang, Z. Ma, X. Yang, C. Wang, Y. Wang, Appl. Surf. Sci. 473, 985 (2019)

38. C. Raju, M. Falmbigl, P. Rogl, P. Heinrich, E. Royanian, E. Bauer, R.C. Mallik, Mater. Chem. Phys. 147, 1022 (2014)

39. Y. Shen, C. Li, R. Huang, R. Tian, Y. Ye, L. Pan, K. Koumoto, R. Zhang, C. Wan, Y. Wang, Sci. Rep. 6, 32501 (2016)

40. A.F. Ioffe, Semiconductor Thermoelements and Thermoelectric Cooling (Infosearch Limited, London, 1957).

41. T. Caillat, A. Borshchevsky, J.P. Fleurial, J. Appl. Phys. 80, 4442 (1996)

42. D.J. Singh, W.E. Pickett, Skutterudite antimonides: quasilinear bands and unusual transport. Phys. Rev. B 50, 11235 (1994)

43. T.M. Tritt, Thermal Conductivity: Theory, Properties, and Applications (Kluwer Academic, New York, 2004).

Publisher's Note Springer Nature remains neutral with regard to jurisdictional claims in published maps and institutional affiliations. 